Dept. of Parasitology and Animal Diseases.

National Research Center, Dokki, Egypt.

\title{
EVALUATION OF IMMUNODIAGNOSTIC ANTIGENS IN TRICHINELLA SPIRALIS MUSCLE LARVAE AND THEIR POTENCY IN DIAGNOSIS OF EARLY INFECTION IN RATS
} (With One Table and 3 Figures)

\section{By}

\section{F.M. EL-MOGHAZY and H.A. SHALABY}

(Received at 23/6/2005)

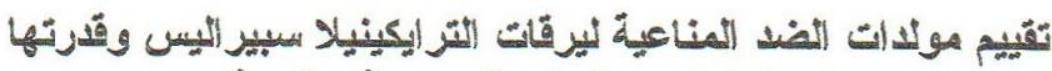 على التشخيص المبكر للفطوى في الجرذان}

\section{فزج الله مهد المغازى ، حاتم عبد الموجود شلبى}

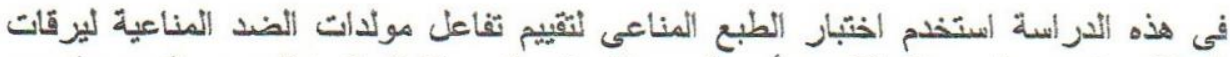

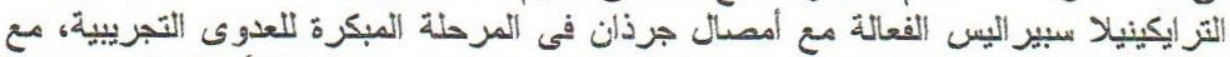

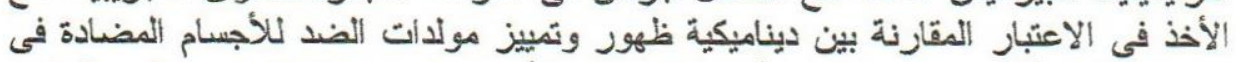

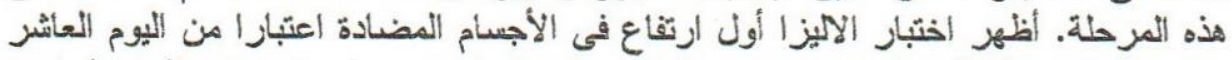

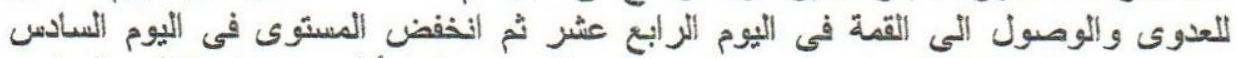

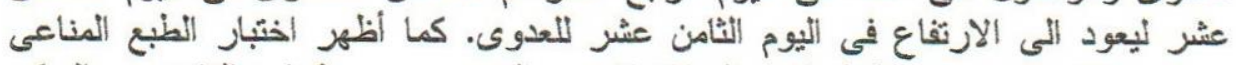

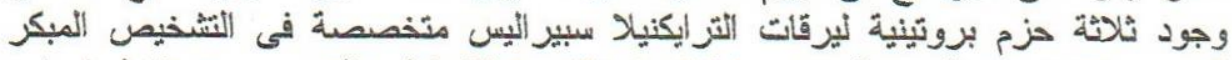

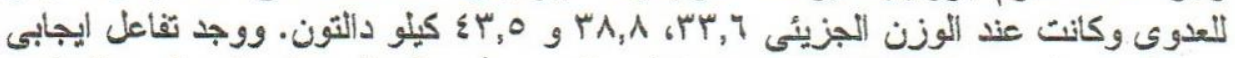

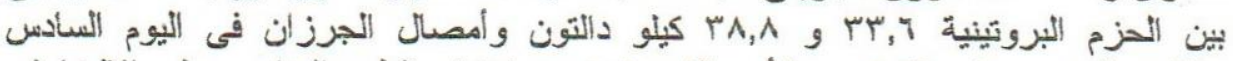

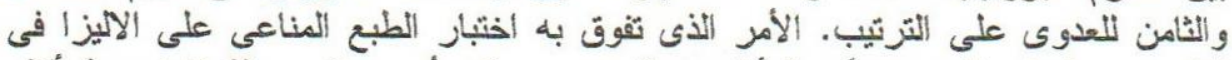

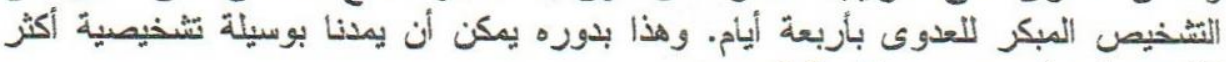

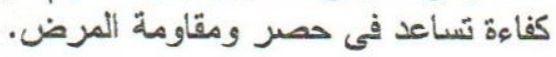

\section{SUMMARY}

An enzyme linked immunotransfer blot assay (EITB) was used in order to characterize reactivity against Trichinella spiralis crude muscle larval antigen (CMLA) in the pooled sera from experimentally infected rats undergoing the early phase of infection. Comparison of the dynamics and antigen recognition profiles of antibodies during the early phase of infection was undertaken in this study. Enzyme-linked immunosorbent assay (ELISA) showed antibody increase on day 10 post-infection (PI). 
The mean antibody levels reached a peak on day 14 PI then decreased on day $16 \mathrm{PI}$ and rose again thereafter on day $18 \mathrm{PI}$. Antigen recognition profile as measured by EITB assay showed three polypeptide bands at molecular weights of $33.6,38.8$ and $43.5 \mathrm{KDa}$ that were specific for the early phase of trichinellosis. EITB could detect the infection four days earlier than ELISA where, two out of the three specific polypeptide bands of $T$. spiralis CMLA $(33.6$ and $38.8 \mathrm{KDa})$ were recognized beginning 6 and 8 days PI, respectively. The advantage that could provide a potentially useful diagnostic tool contributing to surveillance and control of the disease.

Key words: Trichinella spiralis, rats, muscle larval antigen, ELISA, EITB.

\section{INTRODUCTION}

Trichinellosis is one of the food-borne nematode parasitic disease (Eissa et al., 2003) and extensively distributed throughout a wide range of mammalian hosts. From the medical point of view, this disease is primarily a disease of pigs and human. The currently used direct pork inspection procedures (Trichinoscopy or digestion methods) only detected trichinellosis in animals exhibiting relatively severe infections (Gamble, 1996). Besides, it was difficult to detect non-encysted larvae by trichinoscopy (Zimmermann, 1983). That is why there is a worldwide considerable interest in developing a reliable serological test for the detection of antibodies to $T$. spiralis.

In recent years, diagnostic methods for this parasitic infection had been developed in swine and human. These included gel immunodiffusion, indirect immunofluorescence and enzyme immunoassays (Venturiello et al., 1998). ELISA seemed to be the most useful immunodiagnostic test, though reaction specificity was highly dependent on the source of antigen as well as the class of immunoglobulin assayed (Martinez et al., 2000).

One of the many uses of polyclonal antibodies in parasitology is the identification of individual antigens in protein mixtures (Martinez et al., 2000). The spectrum of antibody responses following T. spiralis infection had been examined primarily by immunocytochemistry. This approach revealed localization of antigens at subcellular level, in both larvae and adult worms recognized by sera from infected human, mice and rats (Takahashi et al., 1990). The chronology of antibody response against such antigens suggested that these antigens can be grouped into two sets (Takahashi et al., 1990). The first set was detectable two weeks 
after infection (Rapid-responding). The second set (larval excretory and secretory products) was detectable four weeks after infection (Slowresponding). Each set of antigens seemed to have distinct antigenic profile. Establishment of an assay that have been able to detect the early phase of the infection, the time at which an anti-parasite drug treatment will be most effective, is of interest (Nunez et al., 2003). Therefore, to achieve an early diagnosis of this infection it is important to know the dynamics of synthesis and specificity of immunoglobulins secreted after infection.

Taking the above information into consideration, this work was designed to identify the immunodiagnostic proteins in the $T$. spiralis crude muscle larval antigen which can be used in the early diagnosis of infection.

\section{MATERIALS and METHODS}

\section{Parasites:}

The used strain of $T$. spiralis was originally isolated from diaphragms of infected pigs obtained from Al-Bassatein abattoir, Cairo and has been routinely maintained in the laboratory by consecutive passage through albino rats following the method described by Gamble (1996). Briefly, heavily infected pig diaphragms were minced and digested in $1 \%$ pepsin- $\mathrm{HCl}$. After overnight incubation at $37^{\circ} \mathrm{C}$, larvae were collected using sedimentation technique, washed in physiological saline $(0.85 \%)$ several times and the number of larvae per $\mathrm{ml}$ was counted.

\section{Experimental infection:}

Thirty mature albino rats of $150-200 \mathrm{~g}$ b.wt. were used. They were bred in the Animal Breeding Unit, National Research Center, under controlled light and temperature conditions. Food and water were supplied 'ad libitum'. Rats were divided into two groups. The first group, of twenty rats, was infected orally with 500 larvae / rat. Of them, five rats were sacrificed by cervical dislocation on day 5 PI for detection of adult intestinal phase of $T$. spiralis. While, the remaining rats were sacrificed on day $18 \mathrm{PI}$ for detection of parentral stage of $T$. spiralis. The infected animals were anaesthetized with ether on $2,4,6,8,10,12,14$, 16 and 18 DPI. They were bled from the retro-orbital venous sinus. Blood samples were allowed to clot and then centrifuged at $2000 \mathrm{rpm}$ for 15 minutes. Serum samples from the infected animals at each day post-infection were pooled, aliquoted and stored at $-20^{\circ} \mathrm{C}$ until used. 
The second group; of ten non-infected rats, was served as negative control.

Preparation of $T$. spiralis crude muscle larval antigen (CMLA):

Muscle larvae were obtained by artificial digestion as described before. T. spiralis CMLA was obtained following the method described by Bell and McGregor (1980). The muscle larvae were washed 10 times by sedimentation in $0.01 \mathrm{M}$ phosphate buffer saline (PBS) pH 7.4. After washing, the settled larvae were sonicated for five minutes under 150 watt interrupted pulse cycle using a sonifier cell disrupter. Then they were centrifuged at $10.000 \mathrm{rpm}$ for one hour at $4^{\circ} \mathrm{C}$. The protein content was measured by the method of Lowry et al. (1951) and stored at $-20^{\circ} \mathrm{C}$ until used.

\section{ELISA technique:}

To evaluate the kinetics of appearance of antibodies in the infected rats, during the early phase of infection, versus $T$. spiralis CMLA, ELISA was carried out according to Nunez et al. (2000). The optimal dilutions of various reagents were first determined by checkerboard titrations. Flat-bottomed polystyrene microtitre plate (Linbro, Flow Laboratories, Connecticut, USA) were coated by overnight incubation at $4^{\circ} \mathrm{C}$ with $100 \mu \mathrm{l}$ of $T$. spiralis CMLA diluted to a concentration of $5 \mu \mathrm{g} / \mathrm{ml}$ in $0.1 \mathrm{M}$ sodium carbonate buffer $\mathrm{pH}$ 9.6. After coating and between all subsequent steps, plates were washed 3 times with PBS- $0.05 \%$ Tween 20 (PBS-T). As a blocking reagent, $200 \mu \mathrm{l}$ of a $3 \%$ dry skim-milk solution in PBS were added and incubated for $1 \mathrm{hr}$ at $37^{\circ} \mathrm{C}$. After washing, $100 \mu \mathrm{l}$ of each pooled sera at various periods postinfection diluted 1:400 in PBS-T were added and incubated for $1 \mathrm{hr}$ at $37^{\circ} \mathrm{C}$. After subsequent washing, $100 \mu \mathrm{l}$ of horseradish peroxidasecojugated goat anti-rat IgG (Sigma) diluted 1:1000 in PBS-T were added. After $1 \mathrm{hr}$ incubation, wells were washed again. The color reaction was developed at room temperature by addition of $100 \mu 1$ of $0.04 \%(\mathrm{w} / \mathrm{v})$ ortho-phenylenediamine (Sigma) and $0.004 \%(\mathrm{v} / \mathrm{v}) \mathrm{H}_{2} \mathrm{O}_{2}$ in citrate-phosphate buffer $\mathrm{pH} 5.0$, stopped after $10 \mathrm{~min}$. by adding $1.0 \mathrm{M}$ sulphuric acid and read on an ELISA reader (EL x 800 uv BIO-TEK Instruments INC) at $490 \mathrm{~nm}$. The cutoff $O D$ value previously calculated for this technique was 0.250 , (mean $\pm 2 \mathrm{SD}$ ) of ten non-infected rat sera. Sodium dodecyl sulfate polyacrylamide gel electrophoresis (SDS-PAGE) and EITB techniques:

T. spiralis CMLA was separated by SDS-PAGE in $12 \%$ gels (Laemmli, 1970) in two sets with the aid of wide molecular weight range (Sigma). One set of separated proteins was stained by coomassie 
brilliant blue as a control of native antigen. The other set was used for transference to nitrocellulose paper ( 0.45 um size pore) for EITB assay according to Towbin et al. (1979) to identify the protein fractions of $T$. spiralis CMLA recognized by antibodies present in sera from rats undergoing the early phase of infection $(0,2,4,6,8,10,12,14,16$ and $18 \mathrm{DPI})$. The molecular weight of specific and non-specific polypeptides was determined using Imaging Densitometer (BIO-RAD model GS700).

\section{RESULTS}

Parasitological investigation:

In the experimentally infected rats sacrificed on day $5 \mathrm{PI}$, the numbers of adult worms recovered from the intestine ranged from 200-250 with mean number of $232.4 \pm 10.23$ worm/ rat. Whereas, in the remaining infected rats sacrificed on day 18 PI there was a marked decrease in the number of adult worms recovered from the intestine with mean number of $18.7 \pm 1.5$ worm/ rat. In the meantime, the pepsin digestion technique revealed presence of $1066.67 \pm 32.9$ larvae in the diaphragm and $130 \pm 11.56$ larva/g gastrocnemius muscles.

Occurrence of antibodies in the pooled sera of $T$. spiralis experimentally infected rats at various periods post-infection using ELISA technique:

ELISA showed antibody increase on day 10 PI. The mean antibody levels, that were expressed as mean absorbance values (O.D.490), reached a peak on day 14 PI $(0.87)$ then decreased on day 16 PI (0.52) and rose again thereafter (1.03) on day 18 PI (Fig. 1, Table 1). SDS-PAGE of $T$. spiralis CMLA:

As shown in Fig.(2), T. spiralis CMLA was separated by SDSPAGE into at least 10 protein bands with molecular weight ranging from $14.0-96.0 \mathrm{KDa}$ as measured by densitometry. Of them, 29.7, 33.6, 38.8, 50.4 and $66.7 \mathrm{KDa}$ were major bands.

EITB with pooled sera of non-infected and $T$. spiralis experimentally infected rats at various periods post-infection:

With the EITB, the non-infected rat sera (ODPI) displayed a pattern of non-specific faint reaction with four polypeptide bands, either in doublets or single bands, at $23.8,29.7,66.7$ and $96.0 \mathrm{KDa}$. These polypeptide bands were also recognized with infected rat sera at various periods post-infection. As the infection progressed, the number of recognized bands increased. One additional polypeptide band at molecular weight of $33.6 \mathrm{KDa}$ was recognized beginning 6 days after 
infection. One more doublet band of $38.8 \mathrm{KDa}$ was recognized at $8 \mathrm{DPI}$. A third polypeptide band of $43.5 \mathrm{KDa}$ was the last to be recognized at 14 DPI. As the infection progressed, the reactivity against these bands increased. The recognition of these polypeptide bands; $33.6,38.8$ and $43.5 \mathrm{KDa}$, persisted until the end of experiment (18 DPI) and in the meantime, were not recognized by the non-infected rat sera. Therefore, they seemed to be specific bands for the early phase of trichinellosis.

On comparing the performance of EITB assay with the ELISA technique (Table 1), EITB could detect the infection four days earlier where, the first detectable level of antibodies was recorded beginning 10 DPI by ELISA. As evident from the data in table (1), no correlation could be discerned between the number of specific bands developed by EITB and OD values disclosed by ELISA. Moreover, two out of the three specific polypeptide bands of $T$. spiralis CMLA with molecular weights of 33.6 and $38.8 \mathrm{KDa}$ might consider to be reliable parameter in detecting the early phase of infection earlier than ELISA.

Table 1: Reactivity of pooled sera from $T$. spiralis experimentally infected rats versus $T$. spiralis CMLA at various periods postinfection by ELISA and EITB.

\begin{tabular}{|c|c|c|}
\hline Day post-infection & ELISA $(\mathbf{O D})^{*}$ & EITB $(\mathbf{K D a})^{* *}$ \\
\hline 0 & 0.240 & - \\
2 & 0.240 & - \\
4 & 0.244 & - \\
6 & 0.258 & 33.6 \\
8 & 0.249 & $33.6,38.8$ \\
10 & 0.320 & $33.6,38.8$ \\
12 & 0.337 & $33.6,38.8$ \\
14 & 0.872 & $33.6,38.8,43.5$ \\
16 & 0.518 & $33.6,38.8,43.5$ \\
18 & 1.028 & $33.6,38.8,43.5$ \\
\hline
\end{tabular}

* ELISA cutoff $O D$ value: 0.250

** EITB assay, molecular weight of specific $T$. spiralis CMLA bands. 
Fig. 1: Graph showing the occurrence of antibodies in the pooled sera of $T$. spiralis experimentally infected rats at various periods post-infection.

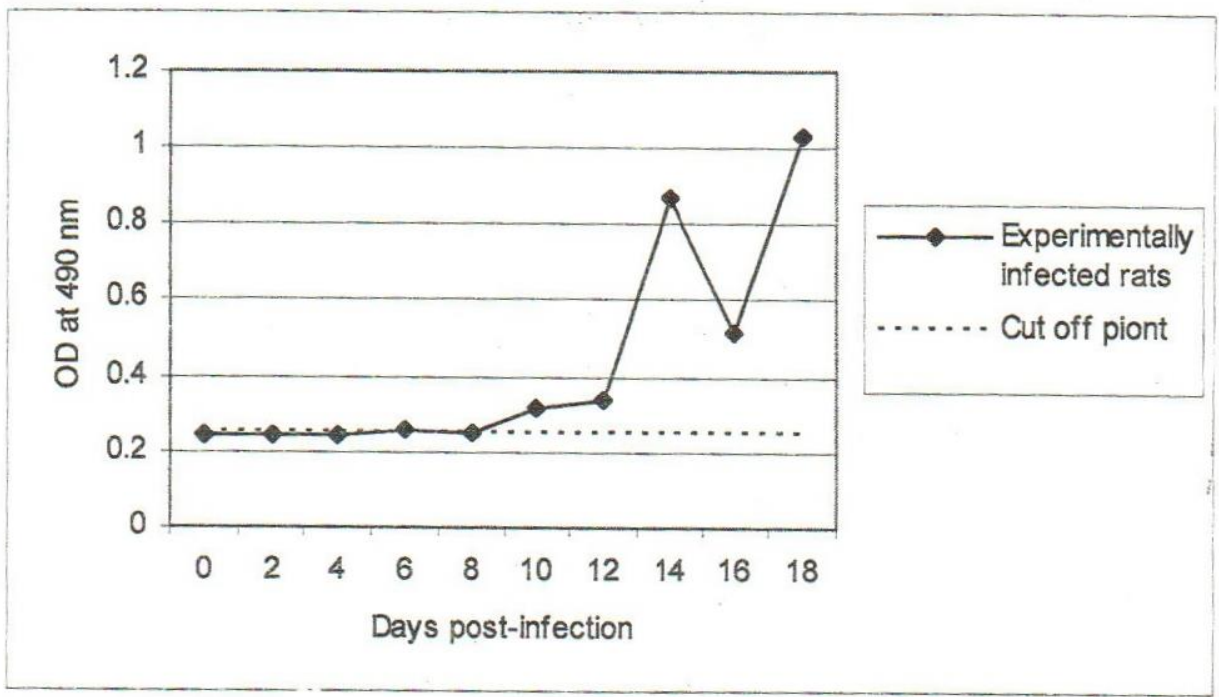

Fig. 2: SDS-PAGE of $T$. spiralis crud muscle larval antigen (CMLA).

MW. Wide molecular weight range.

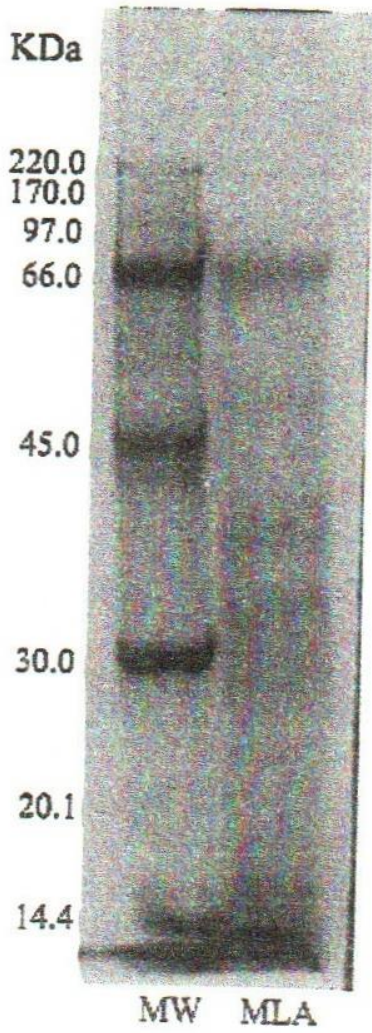




\section{Xid. \\ $9 \%, 0$ \\ 660}

Fig. 3: Antigen recognition on T. spiralis CMLA by antibodies in pooled sera from $T$. spiralis experimentally infected rats at various periods post-infection via EITB assay. The numbers below the lines indicate days PI. Lane numbered 0 correspond to non-infected serum samples. MW. Molecular weight marker stained with Ponceau s solution.

\section{0}

$-43.5 \mathrm{KDg}$

$-38.5 \mathrm{KDe}$

$30.0 \ldots a-33.6 \mathrm{kDs}$

20.1

14.4

\section{DISCUSSION}

In the course of infection, there is a modulation of the host's immune response, depending on the presence of circulating antigens, antigenic presentation, the persistence of antigenic stimulation and the formation of immunocomplexes, among others (Mahanty et al., 1993; Venturiello et al., 1994). Few studies had been carried out on immunomodulatory mechanisms. Most reports evaluated the kinetics of appearance of antibodies without either identifying or characterizing comparatively the antigens recognized by antibodies present in sera from hosts undergoing the early phase of infection (Nunez et al., 2000).

This study aimed to identify serum reactivity against $T$. spiralis CMLA in early phase of infection in rats employing a specific and sensitive assay (EITB) to detect low antibody levels. The performance of EITB assay was compared to ELISA using the same antigen.

The present study revealed successful experimental infection of rats with $T$. spiralis larvae with a mean number of $232.4 \pm 10.23$ adult worms, recovered from the intestine, on day $5 \mathrm{PI}$ and mean larval count of $1066.67 \pm 32.9 \mathrm{1} / \mathrm{rat}$ and $130 \pm 11.56 \mathrm{l} / \mathrm{g}$ recovered from diaphragm and skeletal muscles on day $18 \mathrm{PI}$, respectively. The adult and larval counts as reported herein were in agreement with an earlier observation of the disease in rats (Soliman and El-Bahy 1998). 
The detectable antibody response observed 10 days after infection in ELISA was consistent with the trend observed in mice infected with trichinellosis ( $\mathrm{Li}$ and $\mathrm{Ko}, 2001$ ). The marked increase in its level around day 14 was probably related to the massive migration of newborn larvae as well as nurse cell development (Vassilatis et al., 1992). The subsequent decline in the antibody level was probably caused by the increase of circulating antigens secreted by the larvae and the formation of immunocomplexes. Thereafter, the antibody level rose again on day 18 , when the larva had become fully developed into the infective-stage larva and the encapsulation of the parasite had been completed (Despommier, 1998). Indeed, during this phase in infected mice, a significant amount of circulating antigen was detected as early as days 4 and 6 and reached a peak on day 10 , then declined thereafter as a result of the increase of neutralizing antibodies elicited by the host response ( $\mathrm{Li}$ and $\mathrm{Ko}, 2001)$.

When analyzing the patterns of reactivity obtained with sera from rats with trichinellosis at various periods post-infection as well as from non-infected subjects, it was inferred that the antigenic bands developed at $33.6,38.8$ and $43.5 \mathrm{KDa}$ were specific for the early phase of trichinellosis, regardless of whether the result at each molecular weight was a doublet or single band. It was difficult to compare these results to the one described by other authors (Nunez et al., 2000) owing to the use of different antigenic preparations, conditions of electrophoretic separation and host species. Where, thirteen bands ranging from 14 to $66 \mathrm{KDa}$ were identified in $T$. spiralis excretorysecretory antigen by sera from human with trichinellosis. The bands at approximately $55,36,29$ and $14 \mathrm{KDa}$ proved to be specific. Of them, 55 and $36 \mathrm{KDa}$ bands were a constant feature of serum samples from individuals with the early phase of infection. Moreover, Yera et al. (2003) suggested that the $43-44 \mathrm{KDa}$ and $64 \mathrm{KDa}$ bands of $T$. spiralis CMLA were reactive with all human sera with acute trichinellosis. However, Dupouy-Camet et al. (1988) and other investigators had observed differences in Western blot profiles of sera from humans and animals infected by the same species. In this study, two polypeptide bands at molecular weight of 33.6 and $38.8 \mathrm{KDa}$ of T. spiralis CMLA were recognized beginning 6 and 8 days after infection, respectively. In that sense, Philipp et al. (1981) studied the primary serum antibody response to antigenic surface proteins of infective larvae, intestinal worms and newborn larvae in rats infected with trichinellosis using immuno-precipitation. One week after infection, they found that the sera 
contained antibodies to the surface antigens of both infective larvae and intestinal worms. Furthermore, they concluded that those surface proteins could be targets for antibody-dependent eosinophil-mediated destruction of this parasite. The third polypeptide band of $43.5 \mathrm{KDa}$ was the last to be recognized, in this study, at 14 DPI. This polypeptide band might be related to nurse cell formation. Only a few genes encoding antigens secreted by the larvae had been sequenced and only one of those, the $43 \mathrm{KDa}$ polypeptide had a motif that was suggestive of a function that might be relevant to nurse cell formation (Vassilatis et al., 1992). This protein was synthesized by the alpha stichocytes of the larva and after secretion located exclusively to the nurse cell cytoplasm from day 12 through day 15 of nurse cell development (Vassilatis et al., 1996).

As the infection progressed, the number of recognized bands and the reactivity against these bands increased. The phenomenon could be explained that, at this phase of infection, different adaptive molecules were probably secreted to control and regulate the new habitat (Ko, 1999). Moreover, Ko and Wong (1992) found that excretory-secretory antigens from pre-encapsulated and encapsulated larvae showed different protein profiles and cross-reactivities with heterologous antisera. It appeared that circulating antigen and other antigens secreted by the larvae might play an important role in sustaining the humoral response by stimulating the production of mainly IgG1 antibodies. The development of faint bands from sera of non-infected rats was most likely due to the presence of antigenic epitopes or haptens distributed in nature, e.g., phosphorylcholine among others (Yera et al:; 2003).

On the bases of the results shown in table (1), two out of the three specific polypeptide bands of $T$. spiralis CMLA with molecular weights of 33.6 and $38.8 \mathrm{KDa}$ were considered to be reliable parameter in detecting the early phase of infection earlier than ELISA. These results might be attributable to the low antibody levels encountered at the onset of infection that were readily detected by EITB assay. This observation appeared to be in line with Nunez et al. (2000) who suggested that the EITB assay presented certain advantages over an ELISA that employed the same antigen, because it was not only allowed for the establishment of a specific pattern of reactivity against $T$. spiralis antigen, but it was also capable of detecting. false-negative and borderline ELISA serum samples.

Given the higher sensitivity achieved by the EITB assay during the early phase of infection, it was important to note that the two specific 
polypeptide bands of $T$. spiralis CMLA at molecular weights of 33.6 and $38.8 \mathrm{KDa}$ could detect specific anti-Trichinella antibodies in rats that had recently been infected earlier than ELISA based on the same antigen. The advantage that could provide a potentially useful diagnostic tool contributing to surveillance and control of the disease.

\section{REFERENCES}

Bell, R.G. and McGregor, D.D. (1980): Rapid expulsion of Trichinella spiralis: Co induction by using antigenic extracts of larvae and intestinal stimulation with an unrelated parasite. Infection and Immunity, 29: 194-199.

Despommier, D.D. (1998): How does Trichinella spiralis make itself at home?. Parasitol. Today, 14: 318-323.

Dupoy-Camet, J.; Bougnoux, M.E.; Ancelle, T.; Fagard, R. and Lapierre, J. (1988): Antigenic characteristics of two strains of Trichinella spiralis isolated during the horsemeat-related outbreaks of 1985 in France. Parasitol. Res., 75: 79-80.

Eissa, M.M.; El-Mansoury, S.T. and Allams, S.R. (2003): Coagglutination (Co-A): A rapid test for the diagnosis of experimental trichinellosis. J. Egypt. Soc. Parasitol., 33: 637645.

Gamble, H.R. (1996): Detection of trichinellosis in pigs by artificial digestion and enzyme immunoassay. J. Food Pro., 59: 295-298.

Ko, R.C. (1999): Trichinellosis as a model of new frontier research on parasitic infection. Int. Med. Res. J., 3: 1-11.

Ko, R.C. and Wong, T.P. (1992): Trichinella spiralis: Specificity of ES antigens from pre-encysted larvae. J. Helminthol., 66: 38-44.

Laemmli, U.K. (1970): Cleavage of structural proteins during the assembly of the head of Bacteriophage $T_{4}$. Nature, 227: 680685.

Li, C.K.F. and KO, R.C. (2001): The detection and occurrence of circulating antigens of Trichinella spiralis during worm development. Parasitol. Res., 87: 155-162.

Lowry, O.H.; Rosenbrogh, N.J.; Farr, A.L. and Randall, R.J. (1951): Protein measurement with Folin-phenol reagent. J. Biol. Chem., 193: 265-675. 
Mahanty, S.; King, C.L.; Kumaraswami, V.; Regunathan, J.; Maya, A.; Jayaraman, K.; Abrahams, J.S.; Ottesen, E.A. and Nutman, T.B. (1993): IL-4- and IL-5- secreting lymphocyte populations are preferentially stimulated by parasite-derived antigens in human tissue invasive nematode infections. J. Immunol., 151: 37043711.

Martinez, J.; Perez-Serrano, J.; Bernadina, W.E. and RoddriguezCabeiro, F. (2000): Detection of heat shock protein-70 from $T$. spiralis larvae using a modification of the routine Western blotting procedure. J. Parasitol., 86: 637-639.

Nunez, G.G.; Costantino, S.N. and Venturiello, S.M. (2003): Immunoparasitological parameters of the intestinal phase of trichinellosis in rats. Parasitol., 126: 321-325.

Nunez, G.G.; Malmassari, S.L.; Costantino, S.N. and Venturiello, S.M. (2000): Immunoelectrotransfer blot assay in acute and chronic human trichinellosis. J. Parasitol., 86: 1121-1124.

Philipp, M.; Taylor, P.M.; Parkhouse, R.M.E. and Ogilvie, B.M. (1981): Immune response to stage-specific surface antigens of the parasitic nematode Trichinella spiralis J. Exp. Med., 154: 210215.

Soliman, G.A. and El-Bahy, N.M. (1998): The therapeutic efficacy of doramectin, ivermectin and levamisole against different stages of Trichinella spiralis infection in rats. Vet. Med. J. Giza, 46: 527-541.

Takahashi, Y.; Mizuno, N.; Uno, T.; Aisaka, A. and Araki, T. (1990): A spectrum of antibody response with time after Trichinella spiralis infection in rats. J. Parasitol., 76: 230-239.

Towbin, H.; Stachelin, T. and Gordon, J. (1979): Electrophoretic transfer of proteins from polyacrylamide gels to nitrocellulose sheets. Procedures and some applications. Proc. Nat. Acad. Sci. USA, 76: 4350-4354.

Vassilatis, D.K.; Despommier, D.; Misek, D.E., Polvere, R.I.; Gold, A.M. and Van der Ploeg, L.H.T. (1992): Analysis of a 43-KDa glycoprotein from the interacellular parasitic nematode Trichinella spiralis. J. Biolog. Chem., 267: 18459-18466.

Vassilatis, D.K.; Polvere, R.I.; Despommier, D.D.; Gold, A.M. and Van der Ploeg, L.H. (1996): Developmental expression of a 43-KDa secreted glycoprotein from Trichinella spiralis. Mol. Biochem. Parasitol., 78: 13-23. 
Venturiello, S.M.; Ben, G.J.M.; Costantino, S.N.; Malmassari, S.L.; Nunez, G.G.; Veneroni, R.L. and Traversa, M.J. (1998): Diagnosis of porcine trichinellosis: Parasitological and immunological tests in pigs from endemic areas of Argentina. Vet. Parasitol., 74: 215-228.

Venturiello, S.M.; Gomez, E. and Costantino, S.N. (1994): Eosinophilia and antibody dependent cell cytotoxicity in experimental trichinellosis. In Trichinellosis. Proceedings of the $8^{\text {th }}$ International Conference on Trichinellosis, W.C. Campbell, E. Pozio and F. Bruschi (eds.). Instituto Superiore di Sanita Press, Rome, Italy, PP. 283-288.

Yera, H.; Andiva, S.; Perret, C.; Limonne, D.; Boireau, P. and DupouyCamet, J. (2003): Development and evaluation of a Western blot kit for diagnosis of human trichinellosis. Clin. Dagn. Lab. Immunol., 10: 793-796.

Zimmermann, W.J. (1983): Surveillance in swine and other animals by muscle examination. In: Trichinella and trichinosis. Campbell W.C. ed. New York. Plenum Press. pp. 515-517. 